\title{
CXCR4 is a prognostic marker that inhibits the invasion and migration of gastric cancer by regulating VEGF expression
}

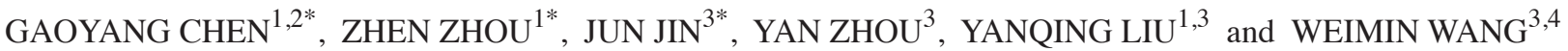 \\ ${ }^{1}$ Department of Chinese Medicine, Nanjing University of Chinese Medicine, Jiangsu, Nanjing 210023; \\ ${ }^{2}$ Department of Oncology, The Second People's Hospital of Taizhou City Jiangsu, Jiangsu, Taizhou 225300; \\ ${ }^{3}$ Institute of Combining Chinese Traditional and Western Medicine, Medical College, Yangzhou University, \\ Jiangsu, Yangzhou 225001; ${ }^{4}$ Department of Oncology, Yixing Hospital Affiliated to Medical College of \\ Yangzhou University, Jiangsu, Yixing 214200, P.R. China
}

Received November 17, 2020; Accepted May 18, 2021

DOI: $10.3892 / \mathrm{ol} .2021 .12848$

\begin{abstract}
Metastasis is the main cause of poor prognosis of patients with gastric cancer (GC). Thus, current research is focused on identifying biomarkers that can predict the prognosis of patients with GC. C-X-C motif chemokine receptor 4 (CXCR4) and vascular endothelial growth factor (VEGF) have been reported to play important roles in different types of malignancies; however, their role in the prognosis of GC remains unknown. The present study aimed to investigate the potential role of CXCR4 and VEGF in predicting the prognosis of patients with GC. Immunohistochemistry analysis was performed to analyze the expression levels of CXCR4 and VEGF in a GC tissue microarray containing GC tissues and adjacent normal tissues. The association between CXCR4 or VEGF expression levels and the clinicopathological characteristics or survival outcomes were assessed. Furthermore, Transwell and wound healing assays were performed to determine the cell invasive and migratory abilities in vitro. The results demonstrated that CXCR4 promoted AGS cell invasion and migration by regulating VEGF expression. In addition, CXCR4 and VEGF expression levels were significantly upregulated in GC tissues compared with adjacent normal tissues, which was associated with a poorer overall survival (OS). Cox regression analysis demonstrated that both
\end{abstract}

Correspondence to: Professor Yanqing Liu, Department of Chinese Medicine, Nanjing University of Chinese Medicine, 138 Xianlin Road, Jiangsu, Nanjing 210023, P.R. China

E-mail: yzliuyq2018@163.com

Professor Weimin Wang, Department of Oncology, Yixing Hospital Affiliated to Medical College of Yangzhou University, 75 Tongzhen Road, Jiangsu, Yixing 214200, P.R. China

E-mail: yzwangweimin@126.com

*Contributed equally

Key words: C-X-C motif chemokine receptor 4, vascular endothelial growth factor, gastric cancer, prognosis, migration, invasion upregulated CXCR4 and VEGF expression were independent negative biomarkers of OS. To the best of our knowledge, the present study was the first to discover that CXCR4 and VEGF exert synergistic roles as efficient prognostic indicators for patients with GC.

\section{Introduction}

Gastric cancer (GC) is one of the most common types of malignancies worldwide (1). Although the incidence of GC has slowly begun to decline, GC remains the third leading cause of cancer-associated mortality worldwide (2). Despite significant improvements being made in the diagnostic and therapeutic methods available for $\mathrm{GC}$, the rate of $\mathrm{GC}$ recurrence remains high, with a 5-year survival rate of $<20 \%$ (3). Metastasis, which is a multi-step process encompassing the proliferation, invasion, detachment, vascular intravasation and adhesion of cancer cells, is the main cause of GC recurrence $(4,5)$. Previous studies have reported a role for numerous cellular molecular markers in the process of metastasis, which will help to guide future research on metastasis $(5,6)$. These should help to identify biomarkers that can be used to predict the prognosis of patients with GC and enable the development of effectvie treatment regimens to improve the survival of patients with GC.

$\mathrm{C}$-X-C motif chemokine receptor 4 (CXCR4), which is a highly conserved member of the $\mathrm{G}$ protein-coupled receptor subfamily, is a transmembrane receptor of 352 amino acids in length. CXCR4 serves as the only receptor for stromal cell-derived factor- 1 and has been reported to play an important role in regulating the differentiation, development and directional migration of immune cells (7). Increasing evidence suggest that CXCR4 expression is upregulated in different types of tumors, where it serves an important role in the occurrence, growth and metastasis of the tumor (8). In addition, CXCR4 was identified as a potential unique molecular target for the diagnosis and treatment of breast (8), lung (9), cervical (10), bladder (11) and colorectal cancer (12). CXCR4 is suggested to serve as a prognostic indicator in patients with $\mathrm{GC}$, which promotes the metastasis of GC $(13,14)$.

Vascular endothelial growth factor (VEGF) is produced by most tumor cells, keratinocytes and macrophages in 
wound sites (15). VEGF receptor ( $\mathrm{R})$ is only expressed on the surface of vascular endothelial cells, and upon binding to its receptor, VEGF can increase vascular permeability, promote the proliferation of vascular endothelial cells, and regulate vasculogenesis and postnatal vascular remodeling (16). VEGF is also known to act as a lymphangiogenic growth factor, serving an important role in tumor lymphangiogenesis via activation of the VEGFRs (17). In addition, signaling pathways associated with VEGF play important roles in the occurrence and development of malignant tumor types (18), including breast cancer (19), hepatocellular carcinoma (20) and lung cancer (21). A VEGF genotype was associated with GC risk in a previous study (22). VEGF protein expression levels in GC tissues were also reported to be positively associated with TNM staging and lymph node metastasis in patients (23).

The present study aimed to determine the expression levels of CXCR4 and VEGF in a cohort of patients with GC and to investigate the potential prognostic and predictive values of these markers in GC. Furthermore, whether detecting the expression levels of CXCR4 and VEGF can be combined as a novel predictor of GC survival with more accuracy than the predictive value of either alone was determined.

\section{Materials and methods}

Patient studies. A total of 589 GC surgical cases were recruited from The Yixing Hospital Affiliated to Medical College of Yangzhou University (Yixing, China) between January 2000 and December 2006. The present study protocol was approved by the Institutional Review Board of Yixing Hospital Affiliated to Medical College of Yangzhou University (approval no. YXYLL-2021-42). All patients provided written informed consent prior to participation and all acquired data were assured of anonymity and confidentiality.

The 589 GC surgical cases were followed up for $\geq 5$ years. Overall survival (OS) was the primary endpoint of the present analysis, and survival time was calculated from the date of surgery to the date of death or to the last follow-up. Detailed clinicopathological characteristics of each patient was obtained from medical records by the ethics committee of the hospital. The clinicopathological characteristics, including age, sex, differentiation stage, depth of invasion, lymph node metastasis, TNM stage (24), distant metastasis and tumor diameter were recorded. All tissue sections were fixed in formalin and embedded in paraffin to construct the tissue microarray (TMA).

A total of 10 paired fresh tissues were immediately frozen in liquid nitrogen following surgical resection and stored at $-80^{\circ} \mathrm{C}$ until subsequent experimentation. The adjacent tissues were all more than $10 \mathrm{~cm}$ away from the cancer tissue. The present study recruited 5 men and 5 women (age range, 35-68 years; mean age, 44 years).

Western blotting. Cells or tissues proteins were extracted using RIPA strong lysis buffer (50 mM pH 7.4 Tris, $150 \mathrm{mM}$ $\mathrm{NaCl}, 1 \%$ Triton $\mathrm{X}-100,1 \%$ sodium deoxycholate, $0.1 \%$ SDS) and $5 \mu \mathrm{l}$ PMSF. The concentration of protein was measured according to the instructions of the BCA kit (Thermo Fisher Scientific, Inc.). Protein samples ( $80 \mu \mathrm{g} /$ lane) were separated via $10 \%$ SDS PAGE, subsequently transferred onto polyvinylidene fluoride membranes (Beyotime Institute of Biotechnology) and blocked with 5\% skim milk at room temperature for $2 \mathrm{~h}$. The membranes were washed with Tris-buffered saline with Tween-20 and incubated with primary antibodies. Western blotting was performed as previously described (25). The following primary antibodies were used: Monoclonal rabbit anti-CXCR4 (1:2,000; cat. no. ab181020; Epitomics; Abcam), monoclonal rabbit anti-VEGF (1:1,000; cat. no. ab32152; Epitomics; Abcam) and monoclonal mouse anti- $\beta$-actin (1:2,000; cat. no. AF5001; Beyotime Institute of Biotechnology). Densitometric analysis was performed using ImageJ software (version 1.44; National Institutes of Health), following normalization to $\beta$-actin expression levels.

Construction of the TMA and immunohistochemistry analysis. CXCR4 and VEGF protein expression levels were analyzed via immunohistochemistry analysis using a formalin-fixed, paraffin-embedded TMA containing samples from patients with GC. The GC TMA included 1,178 cores and each paraffin-embedded tissue sample punched was $1.5 \mathrm{~mm}$ in diameter. These TMAs were heated at $55^{\circ} \mathrm{C}$ for $20 \mathrm{~min}$ and subsequently washed three times with xylene to remove the paraffin. Subsequently, these chips were washed with absolute ethyl alcohol. Antigen retrieval step was performed using sodium citrate and the samples were incubated at $95^{\circ} \mathrm{C}$ for $30 \mathrm{~min}$. Serum blocking was subsequently performed for $30 \mathrm{~min}$ at room temperature. Immunostaining was performed as previously described (25). Briefly, every tissue core was incubated with monoclonal rabbit anti-VEGF (1:200; cat. no. ab32152; Epitomics; Abcam) and monoclonal rabbit anti-CXCR4 (1:200; cat. no. ab181020; Epitomics; Abcam) overnight at $4^{\circ} \mathrm{C}$. The staining scores of the control tissue in each TMA were pre-evaluated as a quality control of the immunostaining.

Evaluation of immunostaining. The staining of CXCR4 or VEGF was evaluated by two independent pathologists who were blinded to the clinical data. The staining results were assessed using a semi-quantitative scoring system, in which the final score was calculated as the product of the proportion and intensity scores. The scoring criteria used for the immunoreactivity score (IRS) was as previously described $(26,27)$. The scoring system used for CXCR4 and VEGF expression involved scoring each sample with a score of between 0 and 12 . The intensity of the immunohistochemistry staining is presented in Fig. 1B and C. The optimum IRS cut-off value was obtained by receiver operator characteristic (ROC) analysis, in which the area under the curve (AUC) at different IRS cut-off values for CXCR4 or VEGF expression was calculated for an OS of 1,3 or 5 years. The optimum cut-off value for the CXCR4 or VEGF IRS was demonstrated to be 5, as it had the best predictive value for survival (Fig. 2A and B). Thus, samples with an IRS of 0-4 were classified as having low CXCR4 or VEGF expression, while samples with an IRS of 6-12 were classified as having high CXCR4 or VEGF expression.

GC cell lines and lentivirus (LV) production. Human AGS GC cells were purchased from The Cell Bank of Type Culture Collection of The Chinese Academy of Sciences. Cells were maintained in RPMI-1640 medium (Hyclone; Cytiva) supplemented with $10 \%$ fetal bovine serum (FBS, Hyclone; Cytiva), at $37^{\circ} \mathrm{C}$ with $5 \% \mathrm{CO}_{2}$. 
A

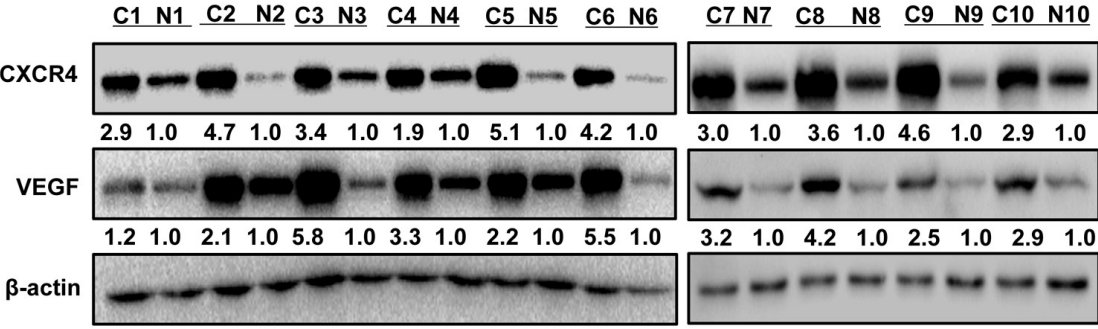

B

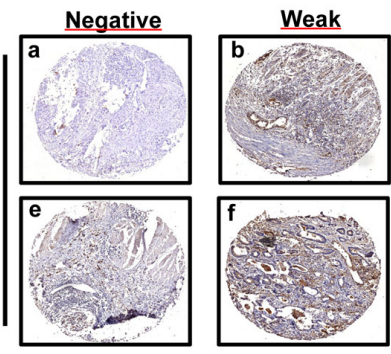

D

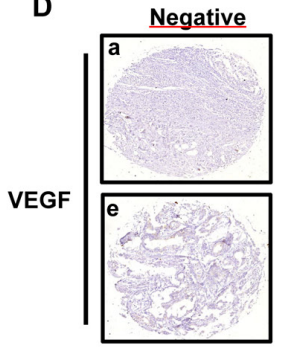

Weak

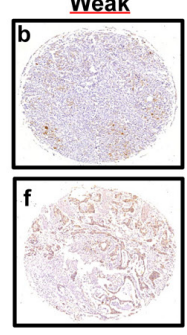

Moderate
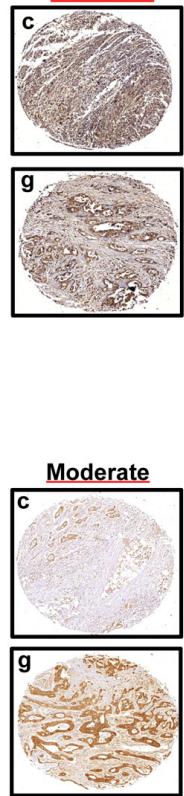
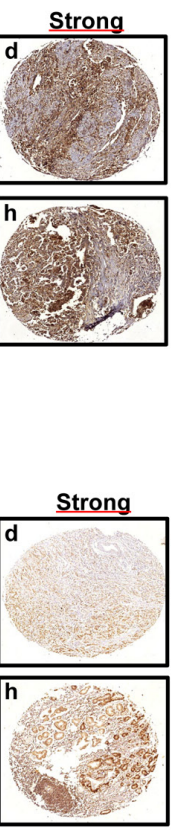

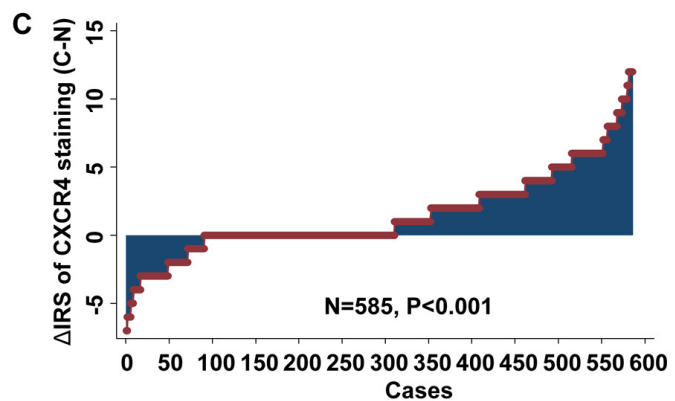

E

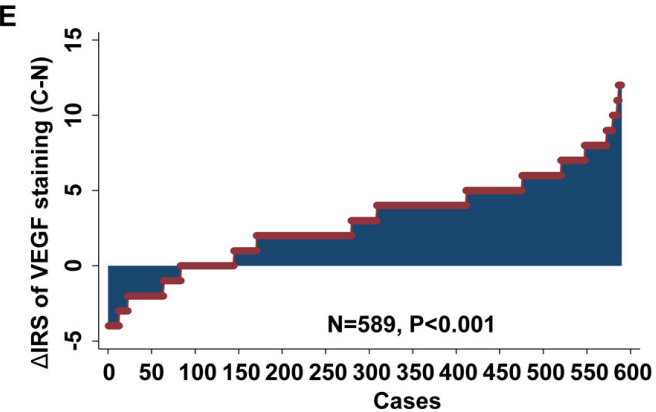

Figure 1. CXCR4 and VEGF expression levels are upregulated in GC. (A) Western blot analysis was performed to detect the protein expression levels of CXCR4 and VEGF, which were upregulated in GC tissues compared with paired adjacent normal tissues. Representative images of (B) CXCR4 or (C) VEGF immunohistochemical staining of the tissue microarray. Panels (B/C-a, B/C-b, B/C-c and B/C-d) exhibit adjacent normal tissues; panels (B/C-e, B/C-d, B/C-g and B/C-h) exhibit GC tissues. Negative staining is presented in panels (B/C-a and B/C-e); weak staining is presented in panels (B/C-b and B/C-f); moderate staining is presented in panels $(\mathrm{B} / \mathrm{C}-\mathrm{c}$ and $\mathrm{B} / \mathrm{C}-\mathrm{g})$ and strong staining is presented in panels $(\mathrm{B} / \mathrm{C}-\mathrm{d}$ and $\mathrm{B} / \mathrm{C}-\mathrm{h})$. Original magnification, $\mathrm{x} 40$. Distribution of (D) CXCR4 and (E) VEGF staining in GC tissues and paired adjacent normal tissues. CXCR4, C-X-C motif chemokine receptor 4; VEGF, vascular endothelial growth factor; GC, gastric cancer; C, cancer; N, normal; IRS, immunoreactivity score.

LV (Shanghai GenePharma Co., Ltd.) was used to interfere with CXCR4 or VEGF expression. LV-CXCR4,LV-CXCR4-RNA interference(RNAi,smallinterferingRNA),LV-VEGF,LV-VEGFRNAi and respective controls were transfected into AGS cells.

Transwell migration and invasion assays. Transwell assays were performed to assess the cell migratory and invasive abilities. Briefly, the membranes of Transwell chambers $(8-\mu \mathrm{m}$ pore size; MilliporeSigma) were pre-coated with Matrigel (MilliporeSigma) for $30 \mathrm{~min}$ for the invasion assay at $37^{\circ} \mathrm{C}$, but not for the migration assay. A total of $100 \mu \mathrm{l} 4 \times 10^{5} / \mathrm{ml}$ cells were subsequently plated in the upper chambers of the Transwell plates and RPMI-1640 medium supplemented with $10 \%$ FBS was plated in the lower chambers. Following incubation for $24 \mathrm{~h}$ at $37^{\circ} \mathrm{C}$, cells in the lower chambers were fixed with methanol for $10 \mathrm{~min}$ and stained with $0.1 \%$ crystal violet for $5 \mathrm{~min}$. Stained cells were counted in five randomly selected fields using an inverted microscope at (x20 magnification).

Wound healing assay. For the wound healing assay, $5 \times 10^{4} / \mathrm{ml}$ cells were seeded into 6 -well plates at $37^{\circ} \mathrm{C}$.
Following incubation, the cell monolayers were scratched using a $10 \mu \mathrm{l}$ pipette tip and cells were cultured in serum-free medium for 0,24 or $48 \mathrm{~h}$. The closure of the wound was visualized in the same field under as inverted ordinary microscope at 20 multiples. The experiments were performed in triplicate.

Statistical analysis. Statistical analyses were performed using SPSS 12.0 software (SPSS, Inc.) and STATA statistical software (version 10.1; StataCorp LP). The Fisher's exact test was used to assess the association between CXCR4 or VEGF expression and the clinicopathological characteristics of patients with GC. Statistical differences in the IRS for CXCR4 or VEGF staining between tumor tissues and paired adjacent normal tissues ( $>5 \mathrm{~cm}$ from the tumor tissue) were determined using the paired Wilcoxon test (raw scores). The correlation between the expression levels of CXCR4 or VEGF was analyzed using the Spearman's rank-order correlation test (raw scores). Univariate and multivariate Cox proportional hazards regression analyses were performed to estimate the crude hazard ratios (HRs), adjusted HRs and 95\% confidence interval (CI) of HRs. The predictive value of CXCR4 and 
A
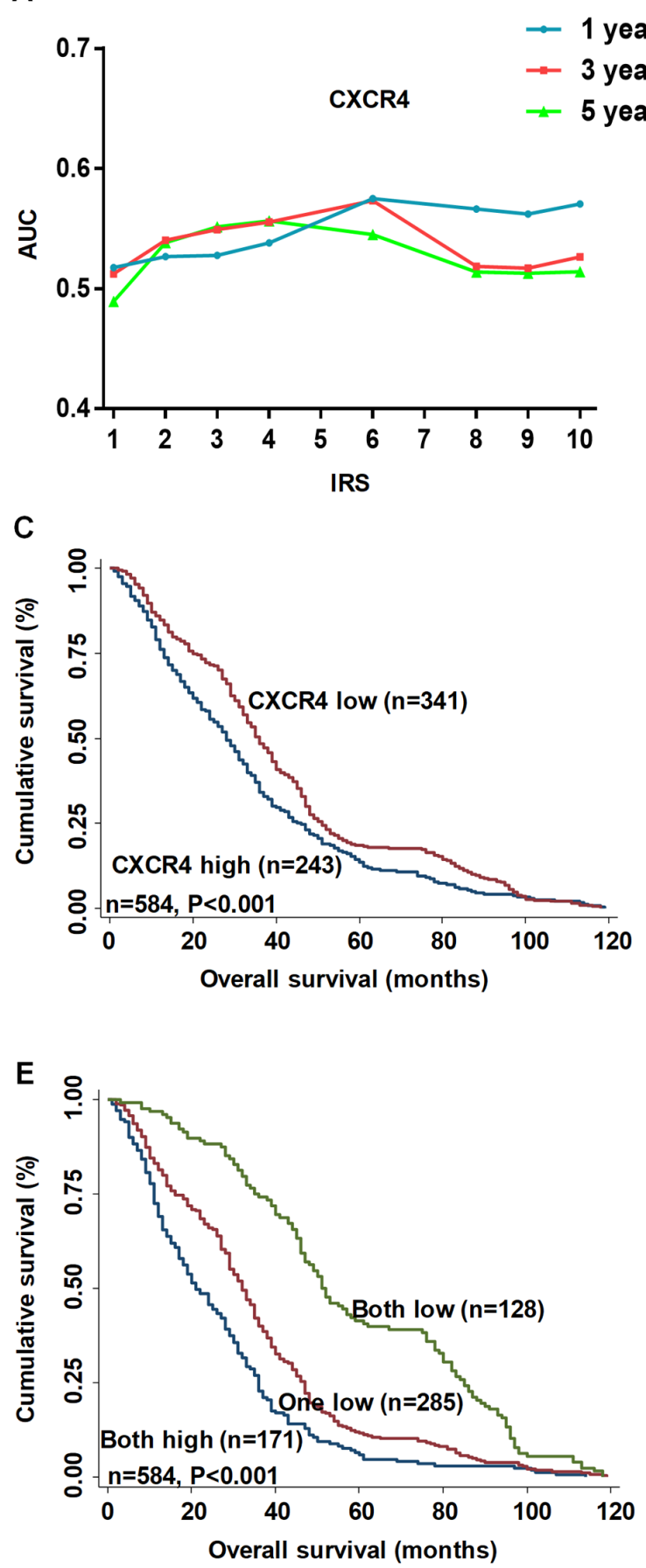

B

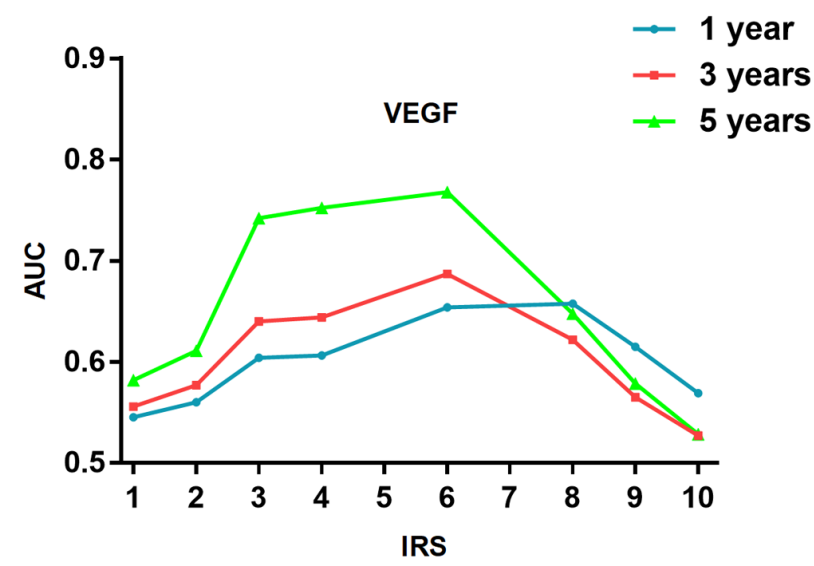

D
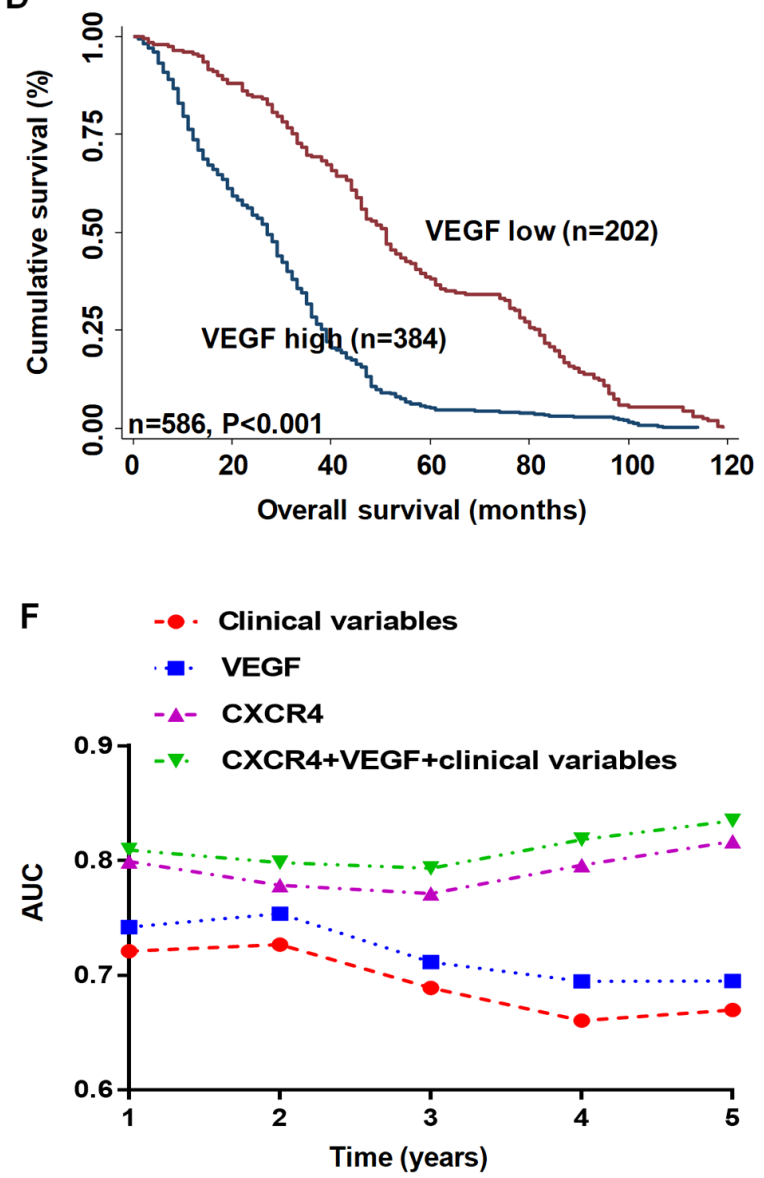

Figure 2. CXCR4 and VEGF expression levels are associated with OS of patients with gastric cancer. (A and B) AUC analysis of different immunoreactivity score cut-off values at 1, 3 and 5 years of OS. Kaplan-Meier survival curves depicting OS according to the expression levels of (C) CXCR4, (D) VEGF and (E) combined CXCR4/VEGF in the training cohort. (F) Time-dependent receiver operator characteristic analyses for clinical risk score (tumor-node-metastasis stage, histological type and tumor diameter), or CXCR4, VEGF or CXCR4 and VEGF risk score. CXCR4, C-X-C motif chemokine receptor 4; VEGF, vascular endothelial growth factor; OS, overall survival; AUC, area under the curve; IRS, immunoreactivity score.

VEGF on OS was evaluated using the Kaplan-Meier method, following by a Weighted Estimation in Cox Regression to determine statistical significance. $\mathrm{P}<0.05$ was considered to indicate a statistically significant difference.

\section{Results}

CXCR4 and VEGF expression levels are upregulated in GC tissues compared with adjacent normal tissues. A total of 10 paired primary GC tissues and matched adjacent normal tissues were used to detect CXCR4 and VEGF protein expression levels via western blotting. The expression levels of CXCR4 and VEGF were upregulated in all tumor tissues compared with the matched adjacent normal tissues (Fig. 1A). The GC TMA slide comprised 589 GC tissues and paired adjacent normal tissues. Analysis of the TMA revealed that CXCR4 and VEGF expression levels were upregulated in tumor tissues compared with paired adjacent normal tissues (Fig. 1B-E). 
Table I. Association between the expression levels of CXCR4 and VEGF and the clinicopathological characteristics of patients with gastric cancer $(n=589)$.

\begin{tabular}{|c|c|c|c|c|c|c|}
\hline \multirow[b]{2}{*}{ Characteristic } & \multicolumn{3}{|c|}{ CXCR4 expression } & \multicolumn{3}{|c|}{ VEGF expression } \\
\hline & Low, n (\%) & High, n (\%) & P-value & Low, n (\%) & High, n (\%) & P-value \\
\hline All patients & $341(58.1)$ & $246(41.9)$ & & $204(34.6)$ & $385(65.4)$ & \\
\hline Age, years & & & 0.125 & & & 0.366 \\
\hline$\leq 65$ & $164(55.6)$ & $131(44.4)$ & & $105(35.5)$ & $191(64.5)$ & \\
\hline$>65$ & $177(60.6)$ & $115(39.4)$ & & $99(33.8)$ & $194(66.2)$ & \\
\hline Sex & & & 0.309 & & & 0.150 \\
\hline Male & $265(58.8)$ & $186(41.2)$ & & $151(33.4)$ & $301(66.6)$ & \\
\hline Female & $76(55.9)$ & $60(44.1)$ & & $53(38.7)$ & $84(61.3)$ & \\
\hline Differentiation stage & & & $0.043^{\mathrm{a}}$ & & & 0.179 \\
\hline $\mathrm{I} / \mathrm{II}$ & $302(56.9)$ & $229(43.1)$ & & $181(34.0)$ & $352(66.0)$ & \\
\hline III & $39(69.6)$ & $17(30.4)$ & & $23(41.1)$ & $33(58.9)$ & \\
\hline Depth of invasion & & & $0.010^{\mathrm{a}}$ & & & $<0.001^{\mathrm{c}}$ \\
\hline $\mathrm{T} 1 / \mathrm{T} 2$ & $122(65.2)$ & $65(34.8)$ & & $101(53.7)$ & $87(46.3)$ & \\
\hline $\mathrm{T} 3 / \mathrm{T} 4$ & $219(54.8)$ & $181(45.2)$ & & $103(25.7)$ & $298(74.3)$ & \\
\hline Lymph node metastasis & & & $<0.001^{\mathrm{c}}$ & & & $<0.001^{\mathrm{c}}$ \\
\hline N0 & $162(72.0)$ & $63(28.0)$ & & $114(50.4)$ & $112(49.6)$ & \\
\hline $\mathrm{N} 1 / \mathrm{N} 2 / \mathrm{N} 3$ & $179(49.4)$ & $183(50.6)$ & & $90(24.8)$ & $273(75.2)$ & \\
\hline TNM stage & & & $<0.001^{\mathrm{c}}$ & & & $<0.001^{\mathrm{c}}$ \\
\hline $\mathrm{I} / \mathrm{II}$ & $186(68.1)$ & 87 (31.9) & & $132(47.9)$ & $142(52.1)$ & \\
\hline III/IV & $155(49.4)$ & $159(50.6)$ & & $72(22.9)$ & $243(77.1)$ & \\
\hline Tumor diameter, cm & & & 0.021 & & & $<0.001^{\mathrm{c}}$ \\
\hline$\leq 5$ & $218(61.6)$ & $136(38.4)$ & & $148(41.7)$ & $207(58.3)$ & \\
\hline$>5$ & $123(52.8)$ & $110(47.2)$ & & $56(23.9)$ & $178(76.1)$ & \\
\hline Distant metastasis & & & $0.002^{\mathrm{b}}$ & & & 0.466 \\
\hline M0 & $330(59.7)$ & $223(40.3)$ & & $193(34.8)$ & $362(65.2)$ & \\
\hline M1 & $11(32.4)$ & $23(67.6)$ & & $11(32.4)$ & $23(67.6)$ & \\
\hline
\end{tabular}

${ }^{\mathrm{a}} \mathrm{P}<0.05 ;{ }^{\mathrm{b}} \mathrm{P}<0.01 ;{ }^{\mathrm{c}} \mathrm{P}<0.001$. CXCR4, C-X-C motif chemokine receptor 4; VEGF, vascular endothelial growth factor; TNM, tumor-node-metastasis

CXCR4 and VEGF expression levels are associated with clinicopathological characteristics. In the TMA containing samples from patients with GC, significant associations were observed between high VEGF expression levels and depth of invasion $(\mathrm{P}<0.001)$, lymph node metastasis $(\mathrm{P}<0.001)$, TNM stage $(\mathrm{P}<0.001)$ and tumor diameter $(\mathrm{P}<0.001)$, using Fisher's exact analysis. However, no significant associations were observed between VEGF expression levels and age, sex, differentiation stage and distant metastasis (Table I).

The two cancer tissues on these chips had fallen off, so only the data of 587 patients were obtained. The Fisher's exact test was also used to assess the association between CXCR4 expression levels and the clinicopathological characteristics of patients with GC. High CXCR4 expression in the GC tissues was significantly associated with differentiation stage $(\mathrm{P}=0.043)$, depth of invasion $(\mathrm{P}=0.010)$, lymph node metastasis $(\mathrm{P}<0.001)$, TNM stage $(\mathrm{P}<0.001)$, tumor diameter $(\mathrm{P}=0.021)$ and distant metastasis $(\mathrm{P}=0.002)$. However, no significant associations were observed between CXCR4 expression and age or sex (Table I).
Upregulated CXCR4 and VEGF expression levels are associated with poor survival in patients with GC. To determine whether CXCR4 or VEGF expression levels are associated with OS in patients with GC, Kaplan-Meier survival curves were used to compare the 5-year overall cumulative survival between patients with high CXCR4 or VEGF staining and patients with low CXCR4 or VEGF staining, respectively. As presented in Fig. 2A and B, low CXCR4 or VEGF expression was 0-4, while high CXCR4 or VEGF expression was 6-12, according to the IRS. The results demonstrated that high expression levels of CXCR4 and VEGF were associated with poor OS in patients with GC (both $\mathrm{P}<0.05$; Fig. $2 \mathrm{C}$ and D). In addition, univariate and multivariate Cox regression analyses were performed to determine whether CXCR4 or VEGF expression levels and the clinicopathological characteristics were associated with the OS of patients with GC. As presented in Table II, depth of invasion, lymph node metastasis, TNM stage, distant metastasis, tumor diameter, and CXCR4 and VEGF expression levels were all statistically significant. Multivariate Cox regression analysis was subsequently 
Table II. Univariate Cox regression analysis of VEGF or CXCR4 expression and clinicopathological characteristics predicting survival in patients with gastric cancer $(n=589)$.

\begin{tabular}{lcr}
\hline Characteristic & HR $(95 \%$ CI $)$ & P-value \\
\hline Age, years ( $\leq 65$ vs. $>65)$ & $1.026(0.872-1.207)$ & 0.761 \\
Sex (male vs. female) & $0.988(0.815-1.199)$ & 0.906 \\
Differentiation stage (I/II vs. III) & $0.817(0.620-1.077)$ & 0.152 \\
Depth of invasion (T1/T2 vs. T3/T4) & $1.858(1.558-2.215)$ & $<0.001^{\mathrm{c}}$ \\
Lymph node metastasis (N0 vs. N1/N2) & $1.917(1.618-2.269)$ & $<0.001^{\mathrm{c}}$ \\
TNM stage (I/II vs. III/IV) & $2.211(1.871-2.612)$ & $<0.001^{\mathrm{c}}$ \\
Distant metastasis (M0 vs. M1) & $1.561(1.102-2.209)$ & $0.012^{\mathrm{a}}$ \\
Tumor diameter, cm ( $\leq 5$ vs. $>5)$ & $1.560(1.321-1.843)$ & $<0.001^{\mathrm{c}}$ \\
CXCR4 expression (low vs. high) & $0.789(0.669-0.931)$ & $0.005^{\mathrm{b}}$ \\
VEGF expression (low vs. high) & $0.391(0.326-0.468)$ & $<0.001^{\mathrm{c}}$ \\
\hline
\end{tabular}

${ }^{\mathrm{a}} \mathrm{P}<0.05 ;{ }^{\mathrm{b}} \mathrm{P}<0.01$; ${ }^{\mathrm{c}} \mathrm{P}<0.001$. VEGF, vascular endothelial growth factor; CXCR4, $\mathrm{C}-\mathrm{X}-\mathrm{C}$ motif chemokine receptor 4; TNM, tumor-node-metastasis; HR, hazard ratio; CI, confidence interval.

performed to assess the effect of CXCR4 or VEGF expression, together with the clinical parameters (age, sex, differentiation stage, depth of invasion and distant metastasis). The results demonstrated that VEGF expression is an independent and unfavorable prognostic factor for patients with $\mathrm{GC}$ ( $\mathrm{HR}, 0.422$; 95\% CI, 0.350-0.508; $\mathrm{P}<0.001$; Table III). Using the same statistical methods, CXCR4 expression was also identified as an independent and unfavorable prognostic factor for patients with GC (HR, 0.836; 95\% CI, 0.708-0.988; P=0.036; Table III).

Synergistic effect of detecting CXCR4 and VEGF expression levels on the $O S$ of patients with $G C$. To determine whether detecting CXCR4 and VEGF expression levels exerts a synergistic effect on predicting the prognosis of patients with GC, Kaplan-Meier survival curves were generated to assess the association between either low CXCR4 and VEGF expression, high CXCR4 and low VEGF expression, low CXCR4 and high VEGF expression or high CXCR4 and VEGF expression, and OS. The results demonstrated that patients with low expression levels of both CXCR4 and VEGF had the most favorable OS amongst the groups $(\mathrm{P}<0.05 ;$ Fig. $2 \mathrm{E})$. Multivariate Cox regression analysis indicated that low expression levels of CXCR4 or VEGF were independent positive prognostic factors for patients with GC (both $\mathrm{P}<0.001$; Table III).

Time-dependent ROC analysis was subsequently performed for the censored data, which indicated that the combination of the clinical risk score (TNM stage, histological type and tumor diameter) and CXCR4 and/or VEGF risk scores was notably higher than either risk score alone in GC TMA cohorts (Fig. 2F). The AUC at 5 years was 0.670 (95\% CI, 0.432-0.671) for the clinical risk score, which significantly increased to 0.852 (95\% CI, 0.527-0.849) when the clinical risk score was combined with both CXCR4 and VEGF risk scores.

CXCR4 promotes AGS cell invasion and migration by regulating VEGF expression. AGS GC cells were infected with LV, and the LV-mediated overexpression or knockdown of CXCR4 or VEGF was analyzed via western blotting (Fig. 3A and B). To determine whether CXCR4 can inhibit AGS cell invasion and migration by regulating VEGF expression, the expression levels of VEGF were altered in AGS cells via lentivirus. As presented in Fig. 3C and D, CXCR4 positively regulated VEGF expression.

As presented in Fig. 3E, the migratory ability of LV-CXCR4transfected AGS cells increased compared with the control group, while the migratory ability of LV-CXCR4-RNAitransfected AGS cells decreased. Similarly, the invasive and migratory abilities of LV-CXCR4-RNAi-transfected AGS cells significantly decreased, whereas the migratory and invasive abilities in LV-CXCR4-transfected AGS cells significantly increased compared with the corresponding control groups ( $\mathrm{P}<0.01$; Fig. 4A and $\mathrm{B})$.

The results of the presents study demonstrated that LV-CXCR4-RNAi-transfected AGS cells had a weaker migratory ability. Following transfection of AGS cells with LV to increase VEGF expression, the migratory ability of LV-CXCR4-RNAi-transfected AGS cells significantly increased $(\mathrm{P}<0.01$; Fig. 4C and D). Conversely, the migratory ability of LV-CXCR4-transfected AGS cells decreased following transfection with LV-VEGF-RNAi ( $\mathrm{P}<0.01$; Fig. 4E and F). Taken together, these results suggest that CXCR4 may promote GC cell migration and invasion by regulating VEGF expression.

\section{Discussion}

During the occurrence and development of GC, cytokines play an important role in the tumor microenvironment by influencing the survival and proliferation of neoplastic and vascular cells $(18,28)$. Previous studies have demonstrated that angiogenic factors are emerging as powerful prognostic tools $(10,29)$. VEGF and matrix metalloproteinase-9 are two of the most important factors involved in the process of angiogenesis (30).

CXCR4 is a crucial member of the chemokine receptor superfamily, and is mainly expressed on granulocytes, T cells, B cells and dendritic cells (7,31). Previous studies have demonstrated that CXCR4 is an important factor associated with human immunodeficiency virus-1 $(14,31,32)$. In addition, some 
Table III. Multivariate Cox regression analysis of CXCR4, VEGF or CXCR4/VEGF expression and clinicopathological characteristics predicting survival in patients with gastric cancer $(n=589)$.

\section{A, CXCR4}

Characteristic $\operatorname{HR}(95 \% \mathrm{CI})$

P-value

Age, years ( $\leq 65$ vs. $>65$ )

$1.016(0.863-1.197)$

0.847

Sex (male vs. female)

$1.081(0.889-1.314)$

0.437

Differentiation stage (I/II vs. III)

$0.852(0.645-1.126)$

0.260

Depth of invasion (T1/T2 vs. T3/T4)

$1.444(1.298-1.606)$

$<0.001^{\mathrm{c}}$

Distant metastasis (M0 vs. M1)

$1.404(0.989-1.994)$

0.058

CXCR4 expression (low vs. high)

$0.836(0.708-0.988)$

$0.036^{\mathrm{a}}$

\section{B, VEGF}

\begin{tabular}{|c|c|c|}
\hline Characteristic & $\operatorname{HR}(95 \% \mathrm{CI})$ & P-value \\
\hline Age, years ( $\leq 65$ vs. $>65)$ & $0.990(0.841-1.165)$ & 0.899 \\
\hline Sex (male vs. female) & $1.125(0.926-1.368)$ & 0.235 \\
\hline Differentiation stage (I/II vs. III) & $0.942(0.713-1.244)$ & 0.675 \\
\hline Depth of invasion (T1/T2 vs. T3/T4) & $1.562(1.302-1.874)$ & $<0.001^{\mathrm{c}}$ \\
\hline Distant metastasis (M0 vs. M1) & $1.736(1.223-2.463)$ & $0.002^{\mathrm{b}}$ \\
\hline VEGF expression (low vs. high) & $0.422(0.350-0.508)$ & $<0.001^{\mathrm{c}}$ \\
\hline
\end{tabular}

\section{C, CXCR4/VEGF}

\begin{tabular}{|c|c|c|}
\hline Characteristic & $\mathrm{HR}(95 \% \mathrm{CI})$ & P-value \\
\hline Age, years ( $\leq 65$ vs. $>65)$ & $1.036(0.880-1.220)$ & 0.671 \\
\hline Sex (male vs. female) & $1.084(0.891-1.318)$ & 0.420 \\
\hline Differentiation stage (I/II vs. III) & $1.014(0.979-1.050)$ & 0.444 \\
\hline Depth of invasion (T1/T2 vs. T3/T4) & $1.635(1.365-1.960)$ & $<0.001$ \\
\hline Distant metastasis (M0 vs. M1) & $1.513(1.068-2.144)$ & $0.020^{\mathrm{a}}$ \\
\hline \multicolumn{3}{|l|}{ CXCR4/VEGF expression } \\
\hline Both low vs. one low & $0.546(0.437-0.681)$ & $<0.001^{\mathrm{c}}$ \\
\hline Both low vs. both high & $0.627(0.552-0.712)$ & $<0.001^{\mathrm{c}}$ \\
\hline
\end{tabular}

${ }^{\mathrm{a}} \mathrm{P}<0.05 ;{ }^{\mathrm{b}} \mathrm{P}<0.01$; ${ }^{\mathrm{C}} \mathrm{P}<0.001$. VEGF, vascular endothelial growth factor; CXCR4, C-X-C motif chemokine receptor 4; TNM, tumor-node-metastasis; HR, hazard ratio; CI, confidence interval.

studies have reported that CXCR4 plays an important role in the process of cancer growth and metastasis $(7,31)$. CXCR4 was suggested to be a potential target for overcoming therapeutic resistance to immune checkpoint blockade in patients with metastatic breast cancer (32). Overexpression of CXCR4 has been demonstrated to be associated with a more advanced tumor stage and poorer survival in patients with GC (14). VEGF expression is widely distributed in various organs and tissues of the body (16). Oncogenes and tumor suppressor genes regulate VEGF by increasing or decreasing its expression (33). Several studies have reported that VEGF expression is closely associated with tumor angiogenesis and plays a key role in tumor growth and metastasis $(18,34)$. Furthermore, previous studies have reported that VEGF expression is upregulated in tumor tissues and the survival rate of patients decreases as VEGF expression increases $(22,35)$. It has also been suggested that
VEGF may be used as a biomarker to predict tumor prognosis in different types of cancer, including lung cancer (36) and breast cancer (19). The present study aimed to determine the association between CXCR4, VEGF and the prognosis of GC.

It is well-known that CXCR4 and VEGF are associated with cancer growth, invasion and metastasis (37). CXCR4 has been reported to promote glioma tumor progression and metastasis via VEGF-mediated angiogenesis (38). In addition, CXCR4 and VEGF-C expression levels are significantly associated with lymph node metastasis in non-small cell lung cancer, and CXCR4 and VEGF-C can synergistically promote metastasis in lung cancer (39). The expression of both CXCR4 and VEGF was also hypothesized to be an effective indicator for predicting the metastatic potential of nasopharyngeal carcinoma (40). In addition, upregulated CXCR4 and VEGF expression levels are associated with increased rates of colon 


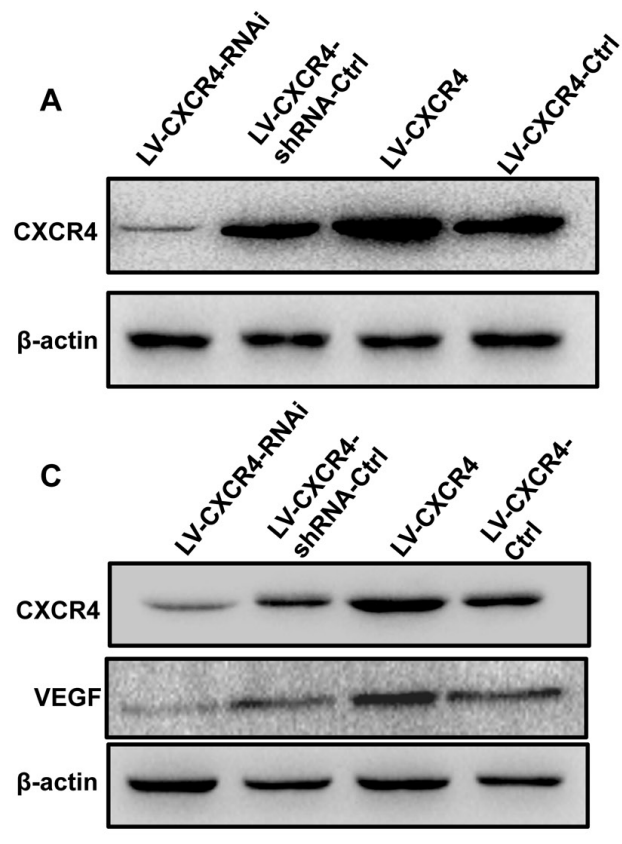

E

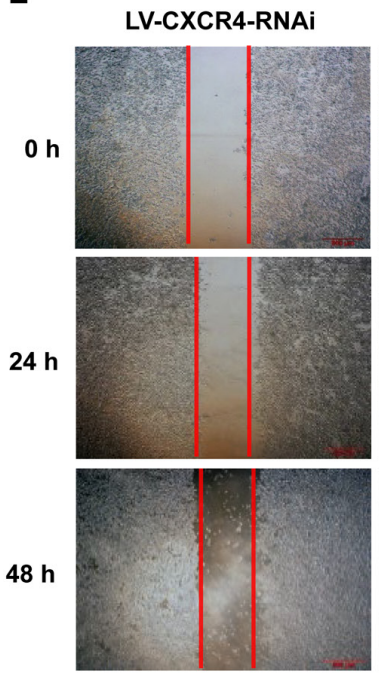

LV-CXCR4-shRNA-CtrI
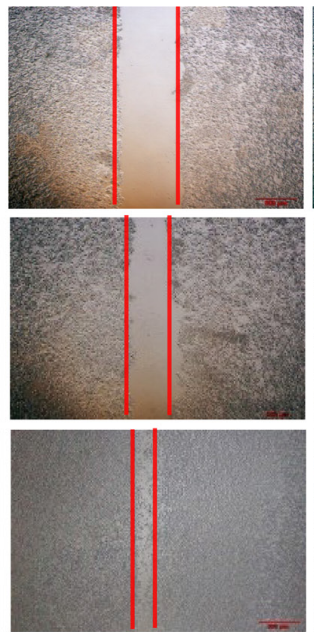
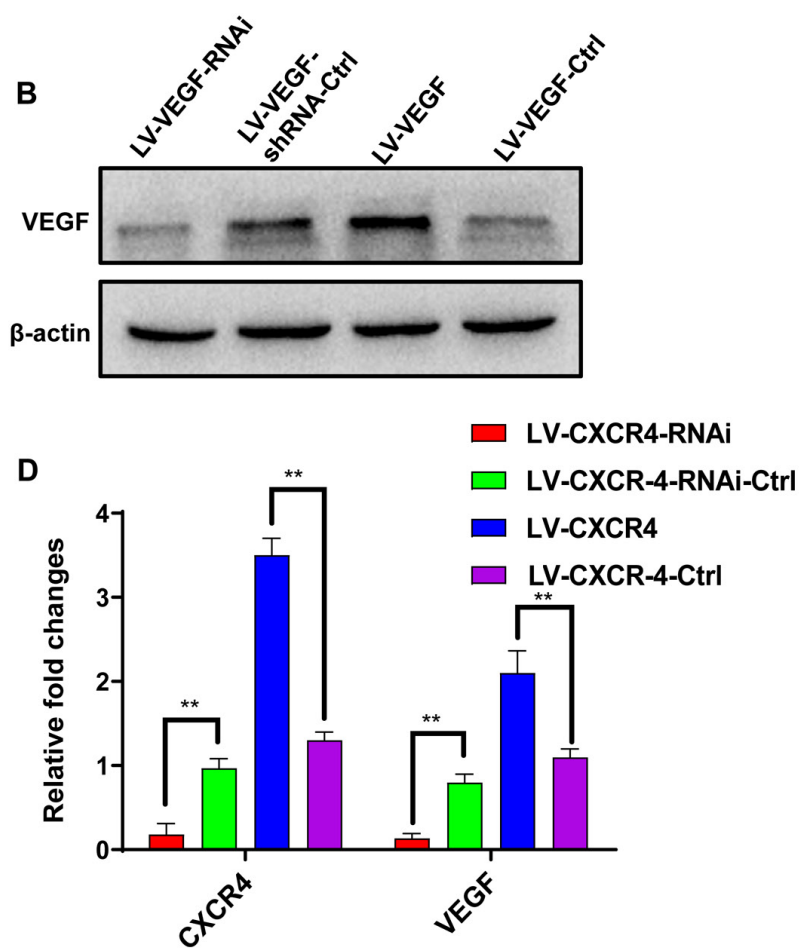

LV-CXCR4

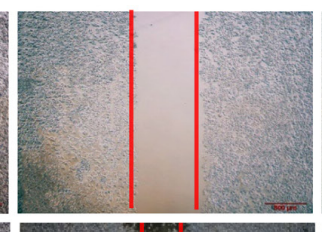

LV-CXCR4-Ctrl

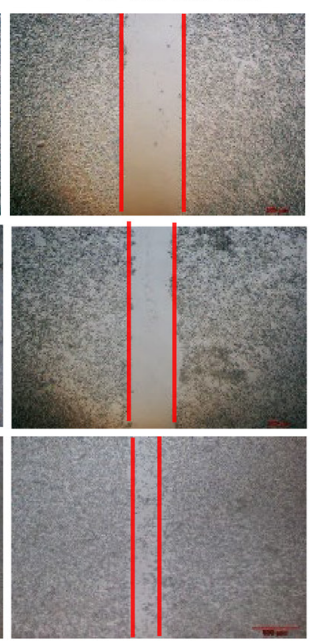

Figure 3. CXCR4 regulates VEGF expression. Protein expression levels of (A) CXCR4 and (B) VEGF were detected via western blotting. (C and D) CXCR4 positively regulated VEGF protein expression levels. (E) The wound healing assay was performed to assess the migratory ability of AGS cells with different CXCR4 expression levels. "* P<0.01. CXCR4, C-X-C motif chemokine receptor 4; VEGF, vascular endothelial growth factor; sh, short hairpin; Ctrl, control.

cancer metastasis (41). The concomitant expression of CXCR4 and VEGF is used as a biomarker for disease-free survival in all patients (41). These findings provide clinical evidence that CXCR4 and VEGF play key roles in GC.

The results of the present study demonstrated that high VEGF expression in GC tissues was significantly associated with the depth of invasion, lymph node metastasis, TNM stage and tumor diameter. In addition, high CXCR4 expression was significantly associated with differentiation stage, depth of invasion, lymph node metastasis, TNM stage, tumor diameter and distant metastasis. Notably, upregulated expression levels of CXCR4 or VEGF were associated with poor OS in patients with GC, as determined by Kaplan-Meier survival analysis. Furthermore, univariate and multivariate Cox proportional hazards regression analyses revealed that both CXCR4 and
VEGF expression were independent negative prognostic factors of GC. In vitro, the expression levels of CXCR4 and VEGF were either overexpressed or knocked down using LV transfection, and the results demonstrated that CXCR4 promoted GC cell invasion and migration by regulating VEGF expression.

The present study also investigated whether the two interacting indicators can be integrated to predict the prognosis of GC more effectively. Through a time-dependent ROC analysis, the results demonstrated that CXCR4 and VEGF expression together had a synergetic effect on predicting the prognosis of patients with GC. Notably, patients with low expression levels of CXCR4 and VEGF had a more favorable survival outcome as demonstrated by Kaplan-Meier survival analysis. 

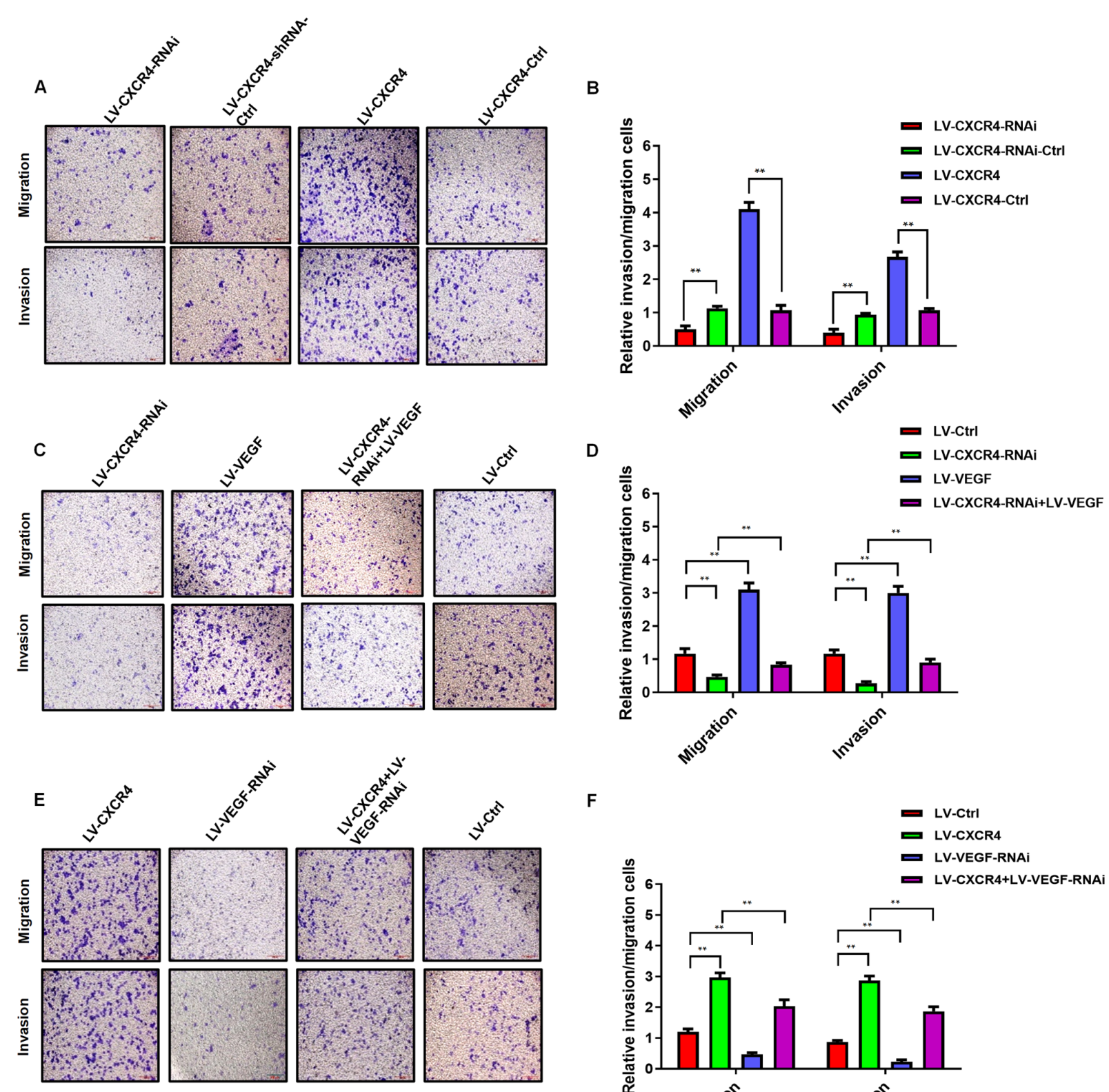

$\mathbf{F}$

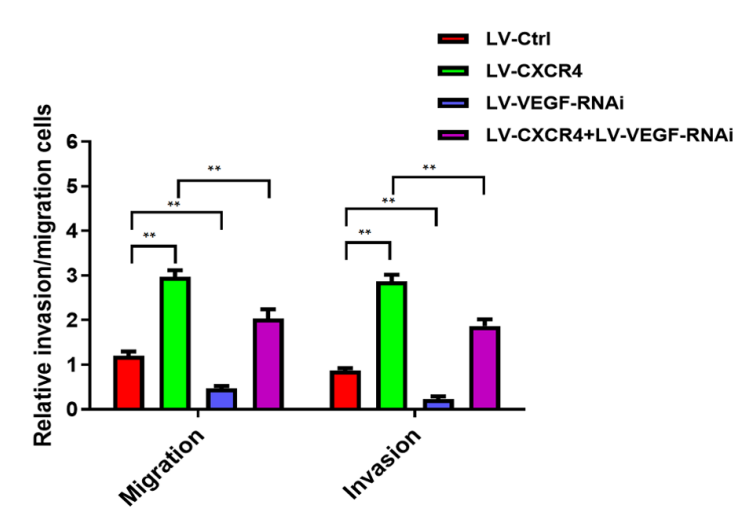

Figure 4. CXCR4 inhibits AGS cell migration and invasion by regulating VEGF expression in vitro. The results of the Transwell assays demonstrated that CXCR4 promoted AGS cell (A) migration and (B) invasion. (C) Invasive and (D) migratory abilities of LV-CXCR4-RNAi-, LV-VEGF-, LV-CXCR4-RNAi + LV-VEGF- and LV-control-transfected AGS cells were analyzed via the Transwell assays. (E and F) Migratory and invasive abilities of LV-CXCR4, LV-VEGF-RNAi, LV-CXCR4+LV-VEGF-RNAi and LV-control transfected AGS cells were analyzed via the Transwell assays. ${ }^{* *} \mathrm{P}<0.01$. CXCR4, C-X-C motif chemokine receptor 4; VEGF, vascular endothelial growth factor; sh, short hairpin; Ctrl, control.

Taken together, these results suggest that CXCR4 or VEGF are both unfavorable prognostic factors for patients with GC. To the best of our knowledge, the present study was the first to investigate the potential of the combined value of CXCR4 and VEGF as efficient prognostic factors for GC. However, further studies are required to verify the functions of CXCR4 and VEGF in other GC cell lines, and determine the molecular mechanisms of these two proteins. Prospective studies will aim to use multicenter samples to validate the results presented here.

\section{Acknowledgements}

Not applicable.

\section{Funding}

The present study was supported by The National Natural Science Foundation of China (grant no. 81773944, awarded to YQL), the Young Medicine Focus Talent Foundation of Jiangsu Province (grant no. QNRC2016206, awarded to WMW), the Postgraduate Research by Practice Innovation Program of Jiangsu Province (grant. no. KYCX18_2382, awarded to WMW), the Top Talent Support Program for young and middle-aged people of Wuxi Health Committee (grant no. 2017, awarded to WMW), the Wuxi City Health Planning Commission project (grant no. MS201815, awarded to WMW; grant no. Z201907, awarded to YZ) and the Natural Science Foundation of Jiangsu Province (grant no. BK20191149, awarded to YZ). 


\section{Availability of data and materials}

The datasets used and/or analyzed during the current study are available from the corresponding author upon reasonable request.

\section{Authors' contributions}

GC, ZZ and JJ contributed to the conception and design of the present study, YZ and JJ retrieved data and drafted the initial manuscript. YL and WW analyzed the data and performed the statistical analysis. GC and ZZ performed the experiments. YL and WW confirmed the authenticity of all the raw data. All authors have read and approved the final manuscript.

\section{Ethics approval and consent to participate}

The present study was approved by the Institutional Review Board of Yixing Hospital Affiliated to Medical College of Yangzhou University (approval no. YXYLL-2021-42) and written informed consent was provided by all participants prior to the study start.

\section{Patient consent for publication}

Not applicable.

\section{Competing interests}

The authors declare that they have no competing interests.

\section{References}

1. Gu ML,Zhou XX, Ren MT, Shi KD, Yu MS, Jiao WR, Wang YM, Zhong WX and Ji F: Blockage of ETS homologous factor inhibits the proliferation and invasion of gastric cancer cells through the c-Met pathway. World J Gastroenterol 26: 7497-7512, 2020.

2. Polkowska-Pruszyńska B, Rawicz-Pruszyński K, Ciseł B, Sitarz R, Polkowska G, Krupski W and Polkowski WP: Liver metastases from gastric carcinoma: A Case report and review of the literature. Curr Probl Cancer 41: 222-230, 2017.

3. Zhao J, Zhao J, Du F, Zhang Y, Shen G, Zhu H, Ji F, Ma F, Dong L, Kan J, et al: Cardia and non-cardia gastric cancer have similar stage-for-stage prognoses after R0 resection: A large-scale, multicenter study in China. J Gastrointest Surg 20: 700-707, 2016.

4. Peinado H, Olmeda D and Cano A: Snail, Zeb and bHLH factors in tumour progression: An alliance against the epithelia phenotype? Nat Rev Cancer 7: 415-428, 2007.

5. Valastyan S and Weinberg RA: Tumor metastasis: Molecular insights and evolving paradigms. Cell 147: 275-292, 2011.

6. Altorki NK, Markowitz GJ, Gao D, Port JL, Saxena A, Stiles B, McGraw T and Mittal V: The lung microenvironment: An important regulator of tumour growth and metastasis. Nat Rev Cancer 19: 9-31, 2019.

7. Huang H, Yuan M, Wu SL, Ba J, Yu X, Mao X and Jin F: Clinical significance of $\mathrm{C}-\mathrm{X}-\mathrm{C}$ motif chemokine receptor 4 and integrin $\alpha v \beta 6$ expression in breast cancer. J Breast Cancer 23: 171-181, 2020.

8. Xu C, Zhao H, Chen $\mathrm{H}$ and Yao Q: CXCR4 in breast cancer: Oncogenic role and therapeutic targeting. Drug Des Devel Ther 9: 4953-4964, 2015.

9. Stumpf C, Kaemmerer D, Neubauer E, Sänger J, Schulz S and Lupp A: Somatostatin and CXCR4 expression patterns in adenocarcinoma and squamous cell carcinoma of the lung relative to small cell lung cancer. J Cancer Res Clin Oncol 144: 1921-1932, 2018.

10. Lecavalier-Barsoum M, Chaudary N, Han K, Koritzinsky M, Hill R and Milosevic M: Targeting the CXCL12/CXCR4 pathway and myeloid cells to improve radiation treatment of locally advanced cervical cancer. Int J Cancer 143: 1017-1028, 2018.
11. Nazari A, Khorramdelazad H and Hassanshahi G: Biological/ pathological functions of the CXCL12/CXCR4/CXCR7 axes in the pathogenesis of bladder cancer. Int J Clin Oncol 22: 991-1000, 2017.

12. Li LN, Jiang KT, Tan P, Wang AH, Kong QY, Wang CY, Lu HR and Wang J: Prognosis and clinicopathology of CXCR4 in colorectal cancer patients: A meta-analysis. Asian Pac J Cancer Prev 16: 4077-4080, 2015.

13. Jiang Q, Sun Y and Liu X: CXCR4 as a prognostic biomarker in gastrointestinal cancer: A meta-analysis. Biomarkers 24: 510-516, 2019.

14. Xiang Z, Zhou ZJ, Xia GK, Zhang XH, Wei ZW, Zhu JT, Yu J, Chen W, He Y, Schwarz RE, et al: A positive crosstalk between CXCR4 and CXCR 2 promotes gastric cancer metastasis. Oncogene 36: 5122-5133, 2017.

15. Albalawi IA, Mir R and Abu Duhier FM: Genetic effects of vascular endothelial growth factor A (VEGF-A) and its association with disease progression in breast cancer population of Saudi Arabia. Asian Pac J Cancer Prev 21: 139-145, 2020.

16. Alagappan VK, Willems-Widyastuti A, Seynhaeve AL, Garrelds IM, ten Hagen TL, Saxena PR and Sharma HS: Vasoactive peptides upregulate mRNA expression and secretion of vascular endothelial growth factor in human airway smooth muscle cells. Cell Biochem Biophys 47: 109-118, 2007.

17. Eroğlu A, Ersöz C, Karasoy D and Sak S: Vascular endothelial growth factor (VEGF)-C, VEGF-D, VEGFR-3 and D2-40 expressions in primary breast cancer: Association with lymph node metastasis. Adv Clin Exp Med 26: 245-249, 2017.

18. Chen C, Chi H, Min L and Junhua Z: Downregulation of guanine nucleotide-binding protein beta 1 (GNB1) is associated with worsened prognosis of clearcell renal cell carcinoma and is related to VEGF signaling pathway. J BUON 22: 1441-1446, 2017.

19. Jain S, Ward MM, O'Loughlin J, Boeck M, Wiener N, Chuang E, Cigler T, Moore A, Donovan D, Lam C, et al: Incremental increase in VEGFR $1 *$ hematopoietic progenitor cells and VEGFR2* endothelial progenitor cells predicts relapse and lack of tumor response in breast cancer patients. Breast Cancer Res Treat 132: 235-242, 2012.

20. Pan Z, Zhuang J, Ji C, Cai Z, Liao W and Huang Z: Curcumin inhibits hepatocellular carcinoma growth by targeting VEGF expression. Oncol Lett 15: 4821-4826, 2018.

21. Naykoo NA, Dil-Afroze, Rasool R, Shah S, Ahangar AG, Bhat IA, Qasim I, Siddiqi MA and Shah ZA: Single nucleotide polymorphisms, haplotype association and tumour expression of the vascular endothelial growth factor (VEGF) gene with lung carcinoma. Gene 608: 95-102, 2017.

22. Liu W, Dong Z, Hu R and Wang C: Association of vascular endothelial growth factor (VEGF) gene polymorphisms with gastric cancer and its development, prognosis, and survival. Technol Cancer Res Treat: Jan 1, 2018 (Epub ahead of print). doi: $10.1177 / 1533034617753810$.

23. Pang L, Wang J, Fan Y, Xu R, Bai Y and Bai L: Correlations of TNM staging and lymph node metastasis of gastric cancer with MRI features and VEGF expression. Cancer Biomark 23: 53-59, 2018.

24. Edge SB and Compton CC: The American Joint Committee on Cancer: The 7th edition of the AJCC cancer staging manual and the future of TNM. Ann Surg Oncol 17: 1471-1474, 2010.

25. Wang S, Wu X, Chen Y, Zhang J, Ding J, Zhou Y, He S, Tan Y, Qiang F, Bai J, et al: Prognostic and predictive role of JWA and XRCC1 expressions in gastric cancer. Clin Cancer Res 18: 2987-2996, 2012.

26. Bai J, Zhou Y, Chen G, Zeng J, Ding J, Tan Y, Zhou J and Li G: Overexpression of Cullin 1 is associated with poor prognosis of patients with gastric cancer. Hum Pathol 42: 375-383, 2011.

27. Wang W, Chen Y, Deng J, Zhou J, Gu X, Tang Y, Zhang G, Tan Y, Ge Z, Huang Y, et al: Cullin1 is a novel prognostic marker and regulates the cell proliferation and metastasis in colorectal cancer. J Cancer Res Clin Oncol 141: 1603-1612, 2015.

28. Pedersen LM, Klausen TW, Davidsen UH and Johnsen HE: Early changes in serum IL-6 and VEGF levels predict clinical outcome following first-line therapy in aggressive non-Hodgkin's lymphoma. Ann Hematol 84: 510-516, 2005.

29. Capone F, Guerriero E, Sorice A, Colonna G, Ciliberto G and Costantini S: Serum cytokinome profile evaluation: A tool to define new diagnostic and prognostic markers of cancer using multiplexed bead-based immunoassays. Mediators Inflamm 2016: 3064643, 2016

30. Zeng XH, Ou ZL, Yu KD, Feng LY, Yin WJ, Li J, Shen ZZ and Shao ZM: Absence of multiple atypical chemokine binders (ACBs) and the presence of VEGF and MMP-9 predict axillary lymph node metastasis in early breast carcinomas. Med Oncol 31: 145, 2014. 
31. Sojane K, Kangethe RT, Chang CC, Moosa MS, Lewin SR, French MA and Ndung'u T: Individuals with HIV-1 subtype C infection and cryptococcal meningitis exhibit viral genetic intermixing of HIV-1 between plasma and cerebrospinal fluid and a high prevalence of CXCR4-using variants. AIDS Res Hum Retroviruses 34: 607-620, 2018.

32. Chen IX, Chauhan VP, Posada J, Ng MR, Wu MW Adstamongkonkul P, Huang P, Lindeman $\mathrm{N}$ and Langer $\mathrm{R}$ and Jain RK: Blocking CXCR4 alleviates desmoplasia, increases T-lymphocyte infiltration, and improves immunotherapy in metastatic breast cancer. Proc Natl Acad Sci USA 116: 4558-4566, 2019

33. Rastegar M, Marjani HA, Yazdani Y, Shahbazi M, Golalipour M and Farazmandfar T: Investigating effect of rapamycin and metformin on angiogenesis in hepatocellular carcinoma cell line. Adv Pharm Bull 8: 63-68, 2018.

34. Honguero Martínez AF, Arnau Obrer A, Figueroa Almazán S, Martínez Hernández N and Guijarro Jorge R: Prognostic value of the expression of vascular endothelial growth factor A and hypoxia-inducible factor 1alpha in patients undergoing surgery for non-small cell lung cancer. Med Clin (Barc) 142: 432-437, 2014.

35. Chou JC, Lieu FK, Ho DM, Shen HY, Lin PH, Hu S, Wang SW, Lin H and Wang PS: Regulation of extracellular and intracellular prolactin on cell proliferation and survival rate through GHR/ JAK2/STAT3 pathway in NSCLC. Chemosphere 264: 128604, 2021.

36. Li H, Takayama K, Wang S, Shiraishi Y, Gotanda K, Harada T, Furuyama K, Iwama E, Ieiri I, Okamoto I, et al: Addition of bevacizumab enhances antitumor activity of erlotinib against non-small cell lung cancer xenografts depending on VEGF expression. Cancer Chemother Pharmacol 74: 1297-1305, 2014.
37. Baci D, Bruno A, Cascini C, Gallazzi M, Mortara L, Sessa F, Pelosi G, Albini A and Noonan DM: Acetyl-L-Carnitine downregulates invasion (CXCR4/CXCL12, MMP-9) and angiogenesis (VEGF, CXCL8) pathways in prostate cancer cells: Rationale for prevention and interception strategies. J Exp Clin Cancer Res 38: 464, 2019.

38. Hong X, Jiang F, Kalkanis SN, Zhang ZG, Zhang XP DeCarvalho AC, Katakowski M, Bobbitt K, Mikkelsen T and Chopp M: SDF-1 and CXCR4 are up-regulated by VEGF and contribute to glioma cell invasion. Cancer Lett 236: 39-45, 2006.

39. Bi MM, Shang B, Wang Z and Chen G: Expression of CXCR4 and VEGF-C is correlated with lymph node metastasis in non-small cell lung cancer. Thorac Cancer 8: 634-641, 2017.

40. Segawa Y, Oda Y, Yamamoto H, Shiratsuchi H, Hirakawa N, Komune S and Tsuneyoshi M: Close correlation between CXCR4 and VEGF expression and their prognostic implications in nasopharyngeal carcinoma. Oncol Rep 21: 1197-1202, 2009.

41. Wu Y, Jin M, Xu H, Shimin Z, He S, Wang L and Zhang Y: Clinicopathologic significance of HIF-1 $\alpha$, CXCR4, and VEGF expression in colon cancer. Clin Dev Immunol 2010: 537531, 2010.

(i) This work is licensed under a Creative Common (c) ${ }_{\text {EY NO ND }}$ Attribution-NonCommercial-NoDerivatives 4.0 International (CC BY-NC-ND 4.0) License. 